\title{
Experimental investigation of fluid flow and heat transfer in a single-phase liquid flow micro-heat exchanger
}

\author{
N. García-Hernando ${ }^{a, *}$, A. Acosta-Iborra ${ }^{a}$, U. Ruiz-Rivas ${ }^{a}$, M. Izquierdo ${ }^{b}$ \\ a Energy Systems Engineering Research Group, Departamento de Ingeniería Térmica y de Fluidos, Universidad Carlos III de Madrid, Avda. de la Universidad 30, \\ Leganés, 28911 Madrid, Spain \\ b Instituto de Ciencias de la Construcción Eduardo Torroja (CSIC), C/Serrano Galvache 4, 28033 Madrid, Spain
}

\begin{abstract}
A B S T R A C T
This work presents an experimental analysis of the hydrodynamic and thermal performance of micro heat exchangers. Two micro heat exchangers, characterized by microchannels of $100 \times 100$ and $200 \times 200 \mu \mathrm{m}$ square cross sections, were designed for that purpose. The fluid used was deionized water and there was no phase change along the fluid circuit. The fluid pressure drop along the heat exchanger and the heat transfer were measured and corrections were made to isolate the contribution of the micro channels. The results were compared with the predictions of the classical viscous flow and heat transfer theory. The main conclusions show that the experimental results fit well with these theories. No effects of heat transfer enhancement or pressure drop increase were observed as a consequence of the small scale of the microchannels.
\end{abstract}

\section{Introduction}

The heat transfer processes between two fluids are common in many engineering systems, such as power plants, chemical reac tors or other power devices. But, while the equipment volume and weight must be constraint, the complexity of such systems is continuously increasing. This is the case, for example, of electronic devices, which should release heat with an increasing capacity per unit area. The direct consequence of these needs is an increasing interest in the miniaturization of heat transfer equipment to obtain micro heat exchangers, where two fluids circulating through chan nels of small cross section (microchannels) interchange heat.

Several investigators have proposed relevant classifications of channels according to their dimension. Tables 1 and 2 show the classifications proposed by Mehendale et al. [1] and Kandlikar and Grande [2]. Both classifications are defined over the value of the smaller dimension of the channels.

The most important parameters of a micro heat exchanger without phase change in practical applications are the frictional losses and the overall heat transfer coefficient. Most of the works published in the literature study and analyze such parameters, the deviations observed from the predictions of classical theory and, when they appear, the mechanisms involved in such deviations.

Already in 1981, Tuckerman and Pease [3] designed and charac terized a micro heat exchanger for the refrigeration of electronic

\footnotetext{
* Corresponding author. Tel.: +34 916248 885; fax: +34 916249430

E-mail address: ngarcia@ing.uc3m.es (N. García-Hernando).
}

devices. The channels were $50 \mu \mathrm{m}$ wide and $300 \mu \mathrm{m}$ deep. They used deionized water and the results obtained showed good agree ment with the classical theory of laminar flow. The thermal resis tance was independent of the mass flow rate when the conditions of fully developed laminar flow were maintained. Later, $\mathrm{Wu}$ and Little [4] focused their efforts on the analysis of pressure drop for channels with equivalent diameters ranging from 50 to $80 \mu \mathrm{m}$. Their results showed that the friction factors obtained were much higher than those predicted by the theory. Since this work, a vast amount of experimental studies have been published with very dif ferent results. In their revisions of previous work, Morini [5] and Steinke and Kandlikar [6] showed that, while some researchers ob serve a general agreement with the classical theory in both heat transfer and pressure drop, others show a small increase of both magnitudes.

The general discrepancies of such results have to be analyzed. To some extent, they can be a consequence of the uncertainties on the measurement process. In this sense, the proper determina tion of the microchannel characteristic diameter is of major impor tance to obtain an accurate friction factor. Also, temperature measurements, with the necessary precision, are difficult to obtain. Another possible explanation is the influence of the entrance re gion, because most of the works consider the flow to be fully devel oped. Moreover, as Steinke and Kandlikar [6] and Kohl et al. [7] show, the inlet and outlet effects should be carefully considered for a proper statement of the pressure drop along the channels. In recent investigations, Chakraborty and co workers [8 10 ], focus on the surface characteristics of microchannels and model the complex mechanisms by which roughness and hydrophobic effects 


\begin{tabular}{|c|c|c|c|}
\hline \multicolumn{4}{|c|}{ Nomenclature } \\
\hline$A$ & elementary conduction area & W & microchannel width \\
\hline$A_{b}$ & microchannel bottom area & $x$ & $x$ coordinate \\
\hline$A_{c}$ & fin cross sectional area & $x^{*}$ & non dimensional microchannel length \\
\hline$A_{g}$ & plate projected area & & \\
\hline$D$ & smaller dimension of the channel & \multicolumn{2}{|c|}{ Greek symbols } \\
\hline$D_{h}$ & hydraulic diameter & $\alpha_{c}$ & microchannel aspect ratio \\
\hline$c$ & specific heat & $\rho$ & density \\
\hline C & heat capacity & $\mu$ & dynamic viscosity \\
\hline$C_{r}$ & heat capacity ratio & $\varepsilon$ & effectiveness \\
\hline$f$ & friction factor & & \\
\hline$H$ & microchannel depth & \multicolumn{2}{|c|}{ Subscripts } \\
\hline$h$ & convective heat transfer coefficient & app & apparent \\
\hline$k_{s}$ & steel thermal conductivity & $c$ & cold flow \\
\hline$L_{m}$ & microchannel length & $c i$ & cold flow inlet \\
\hline$\dot{m}$ & mass flow rate & co & cold flow outlet \\
\hline$N$ & number of microchannels & $\exp$ & experimental \\
\hline $\mathrm{Nu}$ & Nusselt number & $f d$ & fully developed flow \\
\hline $\mathrm{Nu}_{x, 3}$ & local three sided microchannel Nusselt Number & $h$ & hot flow \\
\hline $\mathrm{Nu}_{x, 4}$ & local four sided microchannel Nusselt Number & $h i$ & hot flow inlet \\
\hline$P$ & fin perimeter & ho & hot flow outlet \\
\hline$P_{\text {in }}$ & inlet pressure & $i$ & plate side (hot or cold flow) \\
\hline$P_{\text {out }}$ & outlet pressure & in & inlet \\
\hline $\operatorname{Pr}$ & Prandtl number & ip & inlet plenum \\
\hline$Q$ & heat rate & it & inlet tube \\
\hline $\operatorname{Re}$ & Reynolds number & $m$ & microchannel \\
\hline$R_{\text {fin }}$ & fin thermal resistance & $\max$ & maximum \\
\hline$R_{h}$ & convection thermal resistance & $\min$ & minimum \\
\hline$R_{T}$ & elementary thermal resistance between flows & $o p$ & outlet plenum \\
\hline$t$ & thickness & ot & outlet tube \\
\hline$T$ & temperature & out & outlet \\
\hline$U$ & overall heat transfer coefficient & $p$ & plenum \\
\hline$V$ & velocity & $t$ & tube \\
\hline
\end{tabular}

might alter the fluid flow parameters as compared to classical theory.

Finally, other effects have also been stated in the literature, such as the presence of incondensables (Ghiaasiaan and Laker [11]), the micropolar theory (Papautsky et al. [12]), the viscous dissipation (Tso and Mahulikar [13 15]) and the electrical double layer, EDL (Mala et al. [16], Yang and Li [17,18] and Li [19]). Nevertheless, and as Steinke and Kandlikar [6] stated, all these last factors can be neglected for microchannels with diameters larger than $10 \mu \mathrm{m}$.

This work presents an experimental analysis of the hydrody namic and thermal performance of two micro heat exchangers,

Table 1

Mehendale et al. [1] classification scheme.

\begin{tabular}{ll}
\hline Conventional passages & $D>6 \mathrm{~mm}$ \\
Compact passages & $1 \mathrm{~mm}<D<6 \mathrm{~mm}$ \\
Meso-channels & $100 \mu \mathrm{m}<D<1 \mathrm{~mm}$ \\
Microchannels & $1 \mu \mathrm{m}<D<100 \mu \mathrm{m}$ \\
\hline
\end{tabular}

Table 2

Kandlikar and Grande [2] classification scheme.

\begin{tabular}{ll}
\hline Conventional channels & $D>3 \mathrm{~mm}$ \\
Minichannels & $200 \mu \mathrm{m}<D<3 \mathrm{~mm}$ \\
Microchannels & $10 \mu \mathrm{m}<D<200 \mu \mathrm{m}$ \\
Transitional microchannels & $1 \mu \mathrm{m}<D<10 \mu \mathrm{m}$ \\
Transitional nanochannels & $0.1 \mu \mathrm{m}<D<1 \mu \mathrm{m}$ \\
Nanochannels & $D<0.1 \mu \mathrm{m}$ \\
\hline
\end{tabular}

characterized by microchannels of $100 \times 100$ and $200 \times 200 \mu \mathrm{m}$ square cross sections. The object is to check if the hydrodynamic and thermal behavior agrees with the classical theory in both heat transfer and pressure drop.

\section{Micro-heat exchanger prototypes and experimental facility}

We will focus our characterization in global values for the heat exchanger, measuring the thermal and hydrodynamic characteris tics between the inlet and the outlet, rather than analyzing the individual behavior of the flow in each microchannel.

The design of the experimental facility and its components de pends on the micro heat exchanger dimensions. The microchannel hydraulic diameter and the cross area shape are restricted by the available fabrication technique. Micro mechanization is the tech nique employed, which gives a reasonable mechanization quality for square cross sectional area for channels of at least $100 \mu \mathrm{m}$ in height. It is important to remark the influence that the microfabri cation method and its mechanical characteristics have in the fric tional behavior of liquid microflows. In this sense, Chakraborty et al. [20] have presented a method for evaluating the microchan nel characteristics as a function of the microfabrication process parameters.

Then, the structure and flow configuration of the micro heat exchanger has to be considered. We work with a liquid liquid exchanger without phase change in counter current disposition. A layout of channels at both sides of a plate has been used for efficiency and simplicity purposes. The plate should be thin (as 
possible but restricted to channels mechanization needs) and made of a material with high conductivity, apart of the necessary dimensional stability. The available fabrication facilities and tools restrict the plate thickness to a minimum value of $4 \mathrm{~mm}$. There fore, the microchannels were mechanized over a $4 \mathrm{~mm}$ thick stain less steel plate. Fig. 1 shows a sketch of one of the faces of the plate.

The inlet and outlet flow plenums shown at both sides of the channels are perpendicular to the plate. Seal is obtained using a transparent medium that is placed on each side of the plate to dis tribute the flow and prevent heat losses. The transparent material allows a continuous check of the flow behavior on each microchan nel. Also, the transparent medium acts as a thermal insulator. The geometry of the transparent medium for each side of the plate is shown in Fig. 2. The inlet and outlet are positioned on opposite sides to diminish other heat losses.

Fig. 3(a) shows the design of the micro heat exchanger. The closing is made applying pressure perpendicularly to the transpar ent medium, the metallic plate and the metacrilate square section that surrounds it. Such a closing precludes a hydraulic short circuit between both flows and permits to work without the use of joints. The metacrilate section bears passing holes through it that do not affect the plate.

Two prototypes have been built: one has 100 microchannels of $100 \mu \mathrm{m}$ side and square cross section for each side of the plate and the second has 50 microchannels of $200 \mu \mathrm{m}$ side. Fig. 3(b) shows a picture of the $100 \mu \mathrm{m}$ prototype. The distance between channels was kept equal to the channel side. With these design parameters, both prototypes have the same external dimensions. The number of channels and their length, which is set to $16 \mathrm{~mm}$, are limited by the characteristics of the pump that moves the fluid and the maximum pressure drop available that avoids fluid leakage prob lems (it was fixed around 4 bar). The dimensional dispersion along the channel due to the mechanization process is given in Table 3.

Fig. 4 shows a scheme of the facility designed for the experi ments. Both flows are maintained by a single pump and filter. Reg ulation of the two flows is achieved by the use of two micrometric valves located at the outlet of both currents. The temperature con trol system consists on a tubular electric heater. Only the hot fluid is controlled, while the cold flow enters the exchanger at room temperature. As there is no recirculation, the duration of the exper iments is limited by the supply tank capacity (200 L or 200 min at maximum flow rate).

The measuring system consists of four type $T$ thermocouples that measure the inlet and outlet temperatures of both flows and two differential pressure sensors to measure the pressure drop of each flow along the micro exchanger. The flow rates were inde pendently metered at the discharge zone. Finally, a PC based data acquisition system controls and registers the process variables.

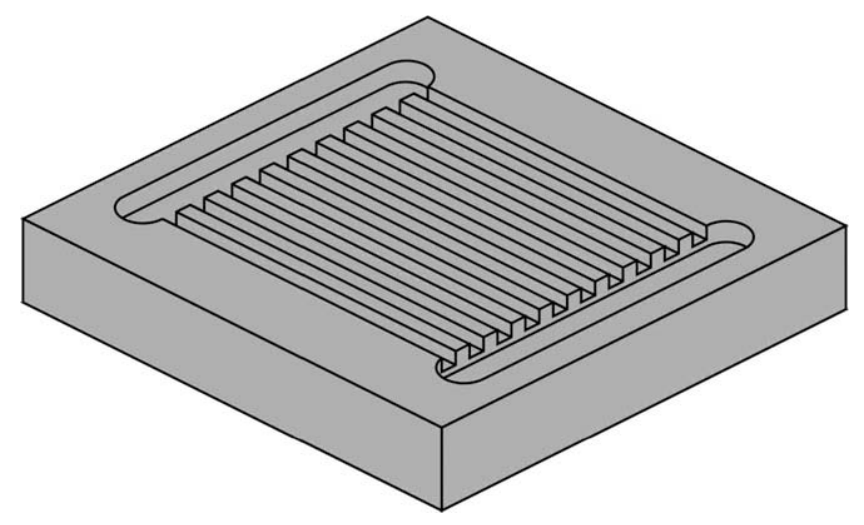

Fig. 1. Layout of the microchannels on the plate.

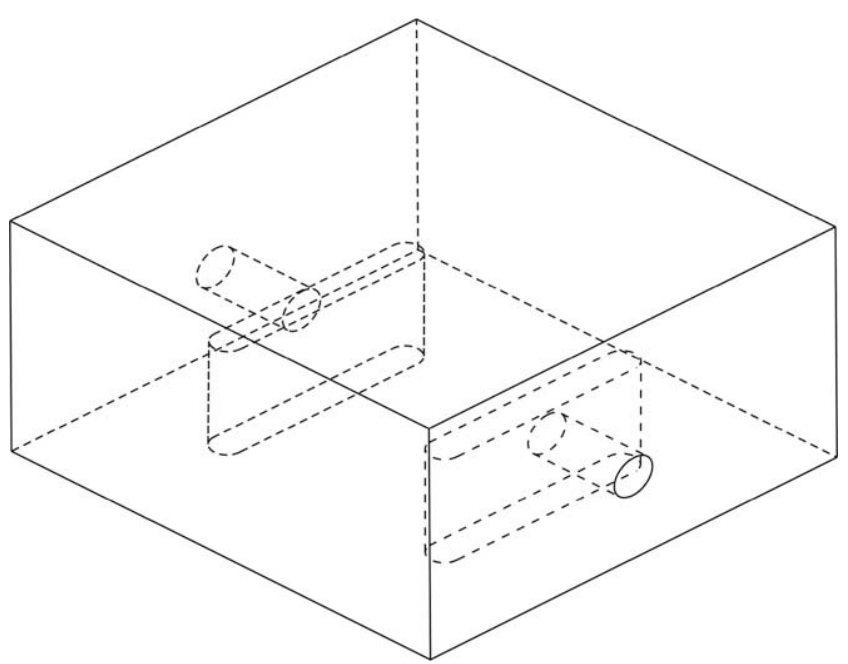

Fig. 2. Layout of the transparent medium.

The thermocouples work between 40 and $125^{\circ} \mathrm{C}$ with a $0.1^{\circ} \mathrm{C}$ error. Three different pressure transducers were employed, depending on the range, up to $5 \mathrm{psi}(0.34 \mathrm{bar})$, up to $30 \mathrm{psi}$ (2.07 bar) and up to 100 psi (6.90 bar). All these transducers have a $1 \%$ full scale error. The flow rates were obtained using a volumet ric method with an overall error of $1 \%$.

Another important source of error is the thermal stability of the laboratory in which the experiments were carried out. Although the heat exchanger is thermally insulated, the change of the room temperature may affect the flow, making difficult to obtain a stea dy state operation. In order to assure the maximum repetitivity, the laboratory was equipped with a climatization system.

\section{Experimental results}

The hydrodynamic and thermal behavior of the two micro heat exchangers is analyzed for a wide range of Reynolds numbers for both currents. The minimum Reynolds number of each current is defined by the minimum flow rate that could be steadily controlled by the pump. The maximum Reynolds number is restricted by the maximum flow rate of the pump, which is $1 \mathrm{~L} / \mathrm{min}$, and occasion ally the pressure drop, that cannot be larger than 4 bar to avoid leaks. As explained in the previous section, both flows can be con trolled independently using the outlet valves, but they are ulti mately linked as they come from the same pump.

We have proceeded in the following manner: once the desired flow rates were established, the heater was turned on to a defined power and after a short lag, a steady state temperature was ob tained in the hot flow inlet of the micro heat exchanger. After wards, also a steady state was obtained in the micro heat exchanger behavior, resulting in steady temperatures at the outlets and a steady pressure drop of both flows. These data were ac quired, and the steady flow rates of both currents measured. Then, the flow rates were changed and the procedure was repeated, con trolling the heater in order to obtain identical steady temperatures in the hot flow inlet. Finally, when the whole range of flow rates had been analyzed, the micro heat inlets were switched (in the side of the plate where the hot fluid was before, flowed the cold fluid and vice versa) and the procedure was repeated.

Along this section and in order to properly state the character istics and origin of each measurement, we will refer to the 100 and $200 \mu \mathrm{m}$ heat exchangers, characterizing the two prototypes by the size of their microchannels and we will talk of side A or B of the plate (which may change from carrying the cold flow or the hot flow). 


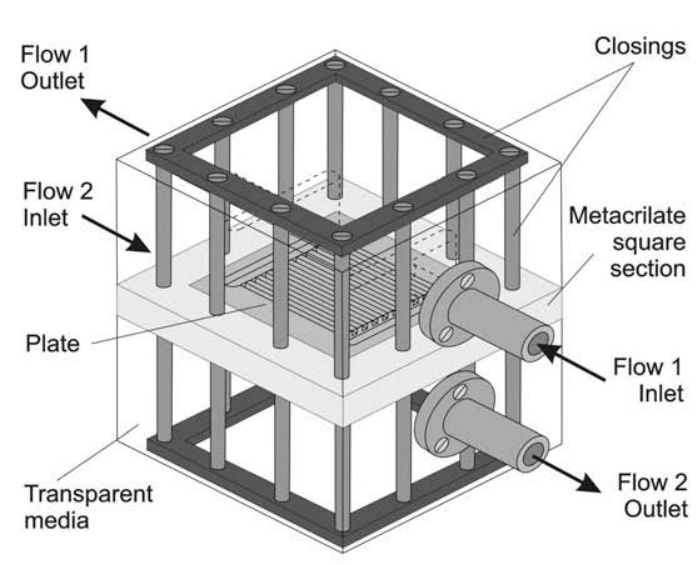

(a)

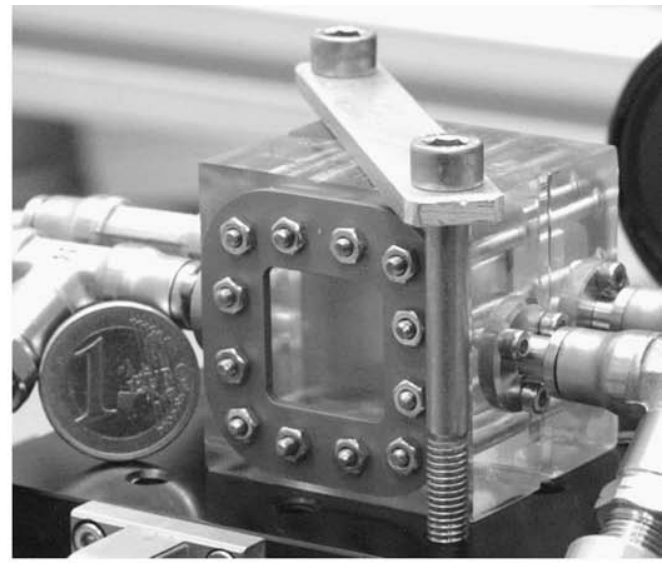

(b)

Fig. 3. Design of the micro-heat exchanger, showing the principal parts and a picture of the $100 \mu \mathrm{m}$ prototype.

Table 3

Dimensional dispersion of the microchannels (Leitz coordinate measuring machine).

\begin{tabular}{|c|c|c|}
\hline & $100 \mu \mathrm{m}$ microchannel & $200 \mu \mathrm{m}$ microchannel \\
\hline \multicolumn{3}{|c|}{ Height dispersion along the channel } \\
\hline Maximum & $\pm 18 \%$ & $\pm 8 \%$ \\
\hline St. deviation & $\pm 6 \%$ & $\pm 5 \%$ \\
\hline \multicolumn{3}{|c|}{ Width dispersion along the channel } \\
\hline Maximum & $\pm 22 \%$ & $\pm 8 \%$ \\
\hline St. deviation & $\pm 12 \%$ & $\pm 4 \%$ \\
\hline \multicolumn{3}{|c|}{ Surface roughness } \\
\hline Maximum & $0.033 \mu \mathrm{m}$ & $0.028 \mu \mathrm{m}$ \\
\hline
\end{tabular}

\subsection{Microchannels pressure drop}

The pressure difference measurements made in the facility do not consist only on the pressure drop along the microchannels, but represent a global pressure drop along the heat exchanger, including the pressure drop also in the two distributors and in the connecting ducts. Therefore, the different sources of pressure drop have to be analyzed separately.

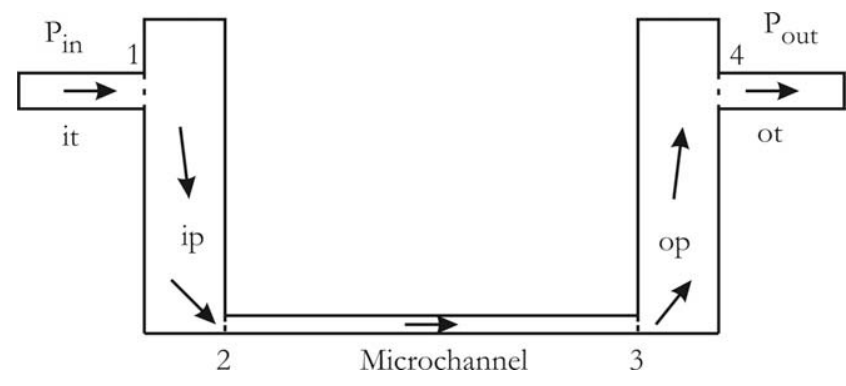

Fig. 5. Longitudinal cut of the heat exchanger, showing the fluid circulation.

Fig. 5 shows a scheme of the fluid circulation inside the heat ex changer. The fluid enters the heat exchanger in Section 1, but the inlet pressure is measured $5 \mathrm{~cm}$ upflow. In Section 1 the flow en ters a plenum chamber and the fluid suffers a sudden expansion, from the $4 \mathrm{~mm}$ duct to the chamber ( $20 \mathrm{~mm}$ wide) and, therefore, the pressure drops. Then, the fluid flows along the chamber and, in Section 2, it enters the microchannels with a sudden contraction from the chamber to a total cross section of the array of

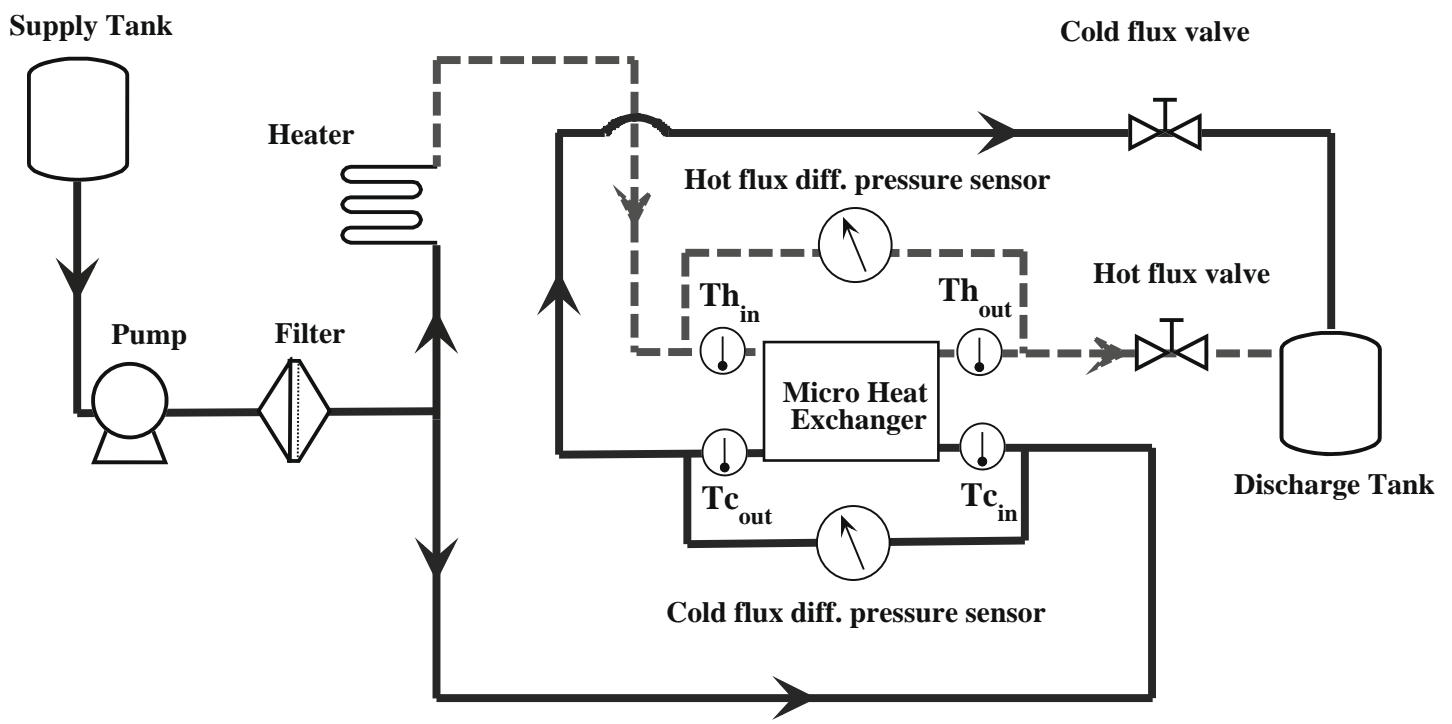

Fig. 4. Scheme of the experimental facility. 
microchannels of 1 or $2 \mathrm{~mm}^{2}$ (depending on the prototype, 100 microchannels of $100 \mu \mathrm{m}$ side or 50 microchannels of $200 \mu \mathrm{m}$ side). The result is another local pressure drop. Finally, the fluid flows through the microchannels and it suffers a sudden expansion at Section 3 and a sudden contraction at Section 4. The changes of flow direction entering and exiting the plenums will also produce pressure drops.

Of all these pressure drops, only the ones produced by the sud den contraction and the sudden expansion at the entrance and the exit of the microchannel, and the pressure drop in the microchan nel are of importance. The fluid velocity is low at the inlet and out let and in the plenums, and the changes of flow direction will cause negligible pressure drops, as the plenum dimension is far larger than the tube or microchannel diameter.

Therefore, the global pressure drop that is measured between inlet and outlet comes from several sources: two expansions ( $\mathrm{Sec}$ tions 1 and 3 ), two contractions (Sections 2 and 4 ), the viscous flow in the inlet and outlet tubes (subscripts it and ot), the viscous flow in the inlet and outlet plenums (subscripts ip and op) and, finally, the viscous flow along the microchannels. This is stated in the fol lowing equation:

$$
\begin{aligned}
\Delta p_{\text {total }} & \Delta p_{\text {microchannel }}+\Delta p_{1}+\Delta p_{2}+\Delta p_{3}+\Delta p_{4}+\Delta p_{i t} \\
& +\Delta p_{i p}+\Delta p_{o p}+\Delta p_{\text {ot }}
\end{aligned}
$$

Eq. (1) can be expanded, using loss coefficients, $K$ (obtained from Kandlikar et al. [21] and supported by recent data obtained by Abdelall et al. [22]), friction factors, $f$ (obtained of well estab lished data and correlations), and the apparent friction factor along the microchannels, $f_{a p p}$, giving Eq. (2):

$$
\begin{aligned}
\Delta p_{\text {total }} \quad & \frac{1}{2} f_{\text {app }} \frac{L_{m}}{D_{h}} \rho_{m} V_{m}^{2}+\frac{1}{2} K_{1} \rho_{1} V_{1}^{2}+\frac{1}{2} K_{2} \rho_{2} V_{2}^{2}+\frac{1}{2} K_{3} \rho_{3} V_{3}^{2} \\
& +\frac{1}{2} K_{4} \rho_{4} V_{4}^{2}++\frac{1}{2} f_{i t} \frac{L_{i t}}{D_{t}} \rho_{i} V_{i t}^{2}+\frac{1}{2} f_{i p} \frac{L_{p}}{D_{p}} \rho_{i} V_{i p}^{2} \\
& +\frac{1}{2} f_{o p} \frac{L_{p}}{D_{p}} \rho_{o} V_{o p}^{2}+\frac{1}{2} f_{o t} \frac{L_{o t}}{D_{t}} \rho_{o} V_{o t}^{2}
\end{aligned}
$$

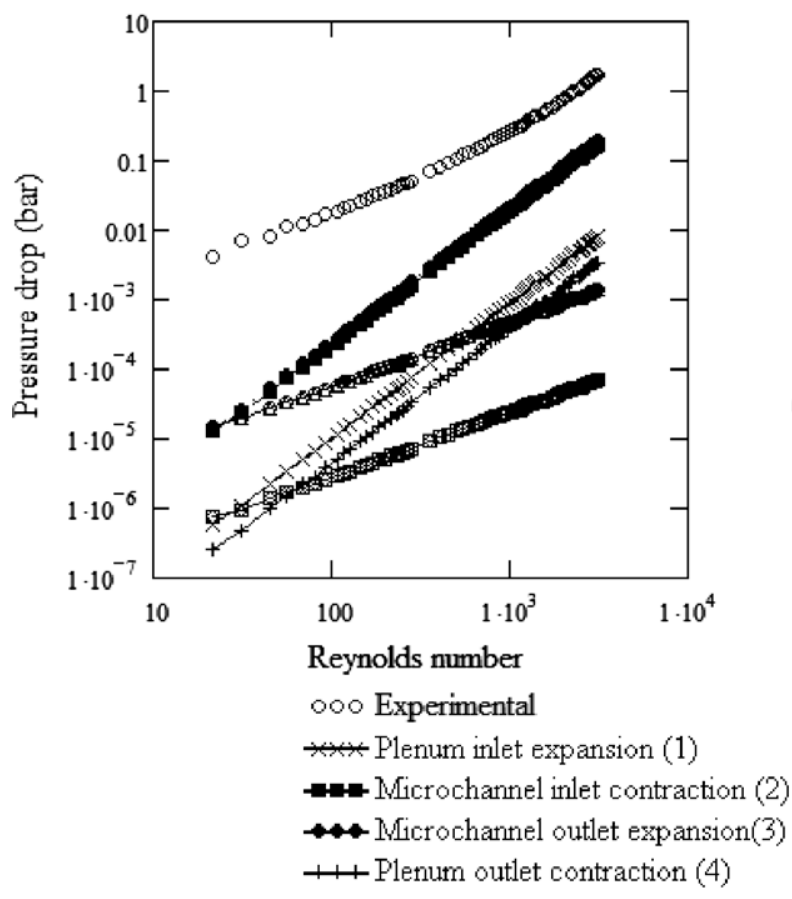

$200 \mu \mathrm{m}$ microchannel where the density, $\rho$, is evaluated before Section 2 at the inlet tem perature, after Section 3 at the outlet temperature and in the micro channels at the average temperature.

All the terms in Eq. (2), apart from the microchannel pressure drop, can be calculated. The results are shown in Fig. 6 for one face of both heat exchangers, showing that only the sudden expansion and contraction when the fluid enters and exits the microchannels are relevant. Quantitative results of both faces of each heat exchan ger are similar when switching from hot to cold fluid or inversely.

Therefore, the pressure drop along the microchannels can be calculated from the measurements, and the microchannel experi mental apparent friction factor obtained:

$f_{\text {app exp }} \frac{2 D_{h}}{L_{m} \rho_{m} V_{m}^{2}} \Delta p_{\text {microchannel }}$

Fig. 7 shows the experimental apparent friction factor obtained from our experiments for both heat exchangers (note that the $x$ axis scale differs in the two graphs in the figure). The experimental results are compared with the analytical apparent friction factor (Eq. (4)) which includes the friction factor of the fully developed flow $\left(f_{f d}\right)$ and the pressure defect or Hagenbach factor, $K(x)$ [23], evaluated in the entire length of the square cross section micro channels, as stated by Steinke and Kandlikar [24] and Kohl et al. [7].

$\frac{2\left(f_{a p p} \mathrm{Re}\right) \mu V x}{D_{h}^{2}} \quad \frac{2\left(f_{f d} \mathrm{Re}\right) \mu V L}{D_{h}^{2}}+\frac{K(x) \rho V^{2}}{2}$

Fig. 8 presents together the data from both heat exchangers. It seems that, for larger Reynolds numbers, the classical theory slightly underestimates the friction factor, but this occurs in the surroundings of the transition to turbulent flow ( $\operatorname{Re}>2300)$. In this region, the dimensional uncertainties shown in Table 3, and the dimensional dispersion inherent to the microfabrication procedure (not only between different sides but even between microchannels of the same side) might be of major importance, and especially in the $100 \mu \mathrm{m}$ heat exchanger, in which the effect is clearer. There
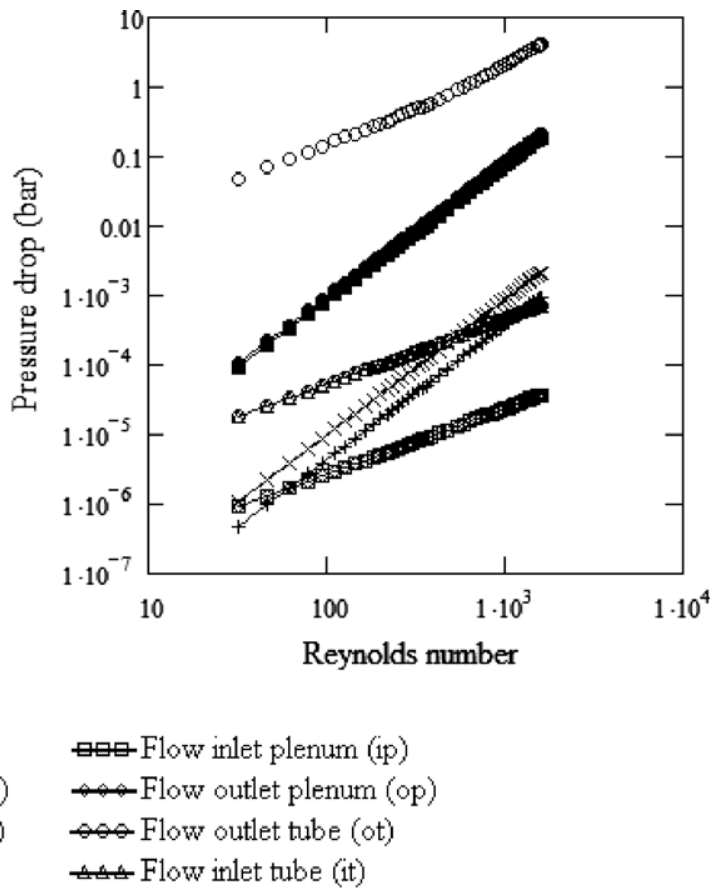

$100 \mu \mathrm{m}$ microchannel

Fig. 6. Pressure drop along the heat exchanger. 


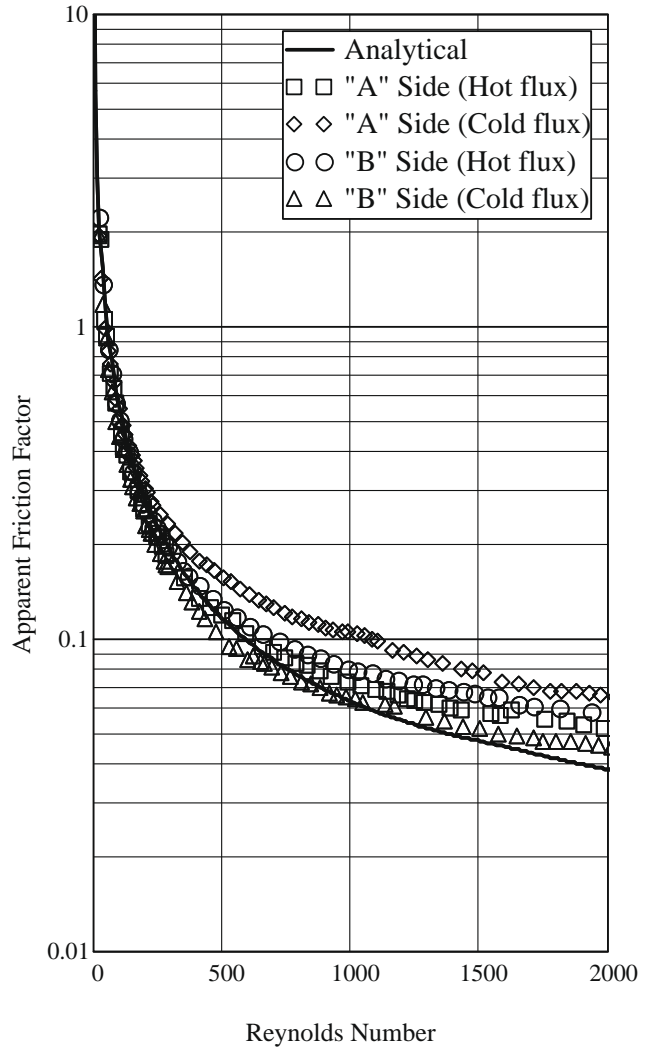

$200 \mu \mathrm{m}$ microchannel

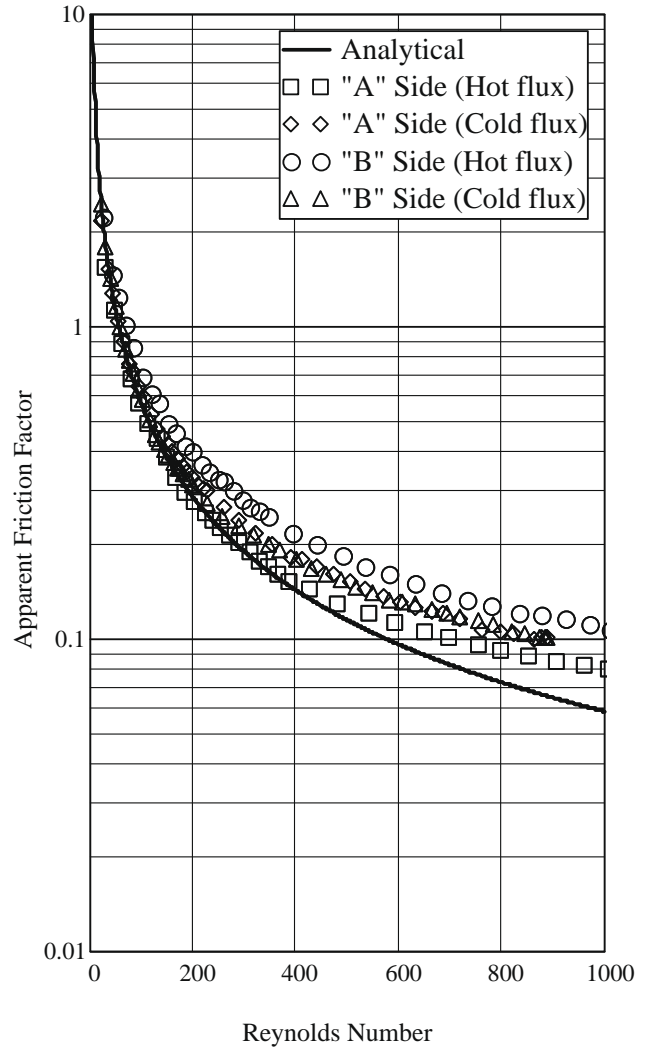

$100 \mu \mathrm{m}$ microchannel

Fig. 7. Experimental and analytical apparent friction factors.

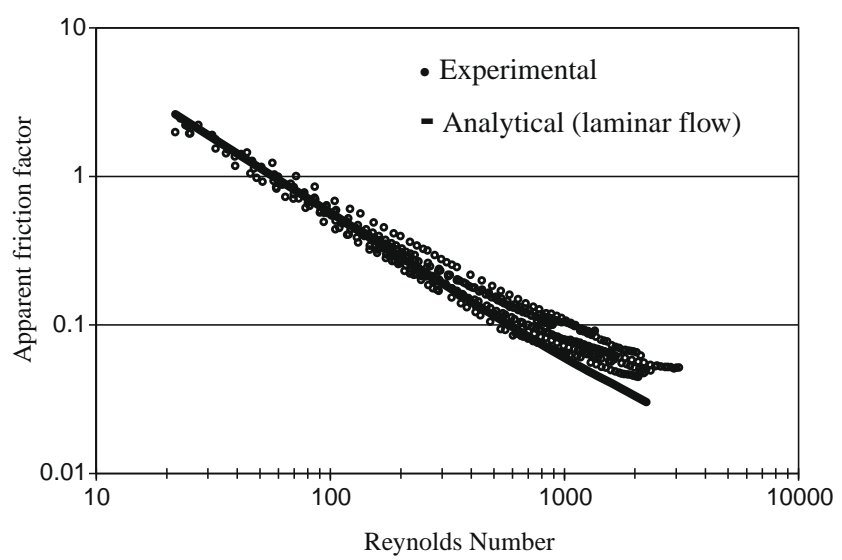

Fig. 8. Comparison of experimental and analytical apparent friction factors for both heat exchangers.

fore, the experimental results permit to conclude that a general good agreement of the microchannels pressure drop with conven tional laminar flow theory (considering entrance effects by means of the Hagenbach factor) is obtained and no significant deviation appears.

\subsection{Heat transfer}

\subsubsection{Experimental results}

The heat transferred by the micro heat exchanger, $Q_{i}$, can be calculated from the measured temperatures and flow rates:

$Q_{i} \quad \dot{m}_{i} c_{i}\left(T_{\text {in }} \quad T_{\text {out }}\right)_{i}$ where the subscript $i$ refers either to the hot or cold flow, the mass flow rate $\dot{m}$ is calculated from the measured volumetric flow rate and $c$ is the specific heat evaluated at the average temperature in the channel.

Fig. 9 shows the results obtained in our experiments for the two micro heat exchangers. Graphs (a) show the pair of Reynolds num bers (for the cold and the hot flow) used in each experiment, de fined to get approximately equal mass flow rates on both flows (and therefore similar temperature increments). Graphs (b) and (c) show the heat transferred from the hot flow and to the cold flow, respectively. Finally, graphs (d) show the difference between these two terms, including both heat losses and measurement errors.

In this last figure, the $x$ axis represents the measurement case number and the experiments where numbered for increasing Reynolds number (notice that each point represents an experi mental condition given by a pair of different Reynolds numbers, for hot and cold fluids, as stated in the previous paragraph). The maximum relative error is around $15 \%( \pm 2 \mathrm{~W})$, and it can be lar gely attributed to the measurement system accuracy. It might be argued that heat losses to the ambient air may have an inci dence in this value. In this sense, Li et al. [25] already stated, the heat losses at low Re conditions demand particular attention because their value might become important compared to the heat effectively transferred between fluids. In our case, special care has been taken to minimize those losses. The isolation of the heat exchanger (built on metacrilate, with thermal conduc tivity lower than $0.2 \mathrm{~W} / \mathrm{m}^{2} \mathrm{~K}$ ) and the isolation of the inlet and outlet conductions (elastomeric insulation with thermal conduc tivity lower than $0.04 \mathrm{~W} / \mathrm{m}^{2} \mathrm{~K}$ ) makes it difficult to consider this the source of such errors. 

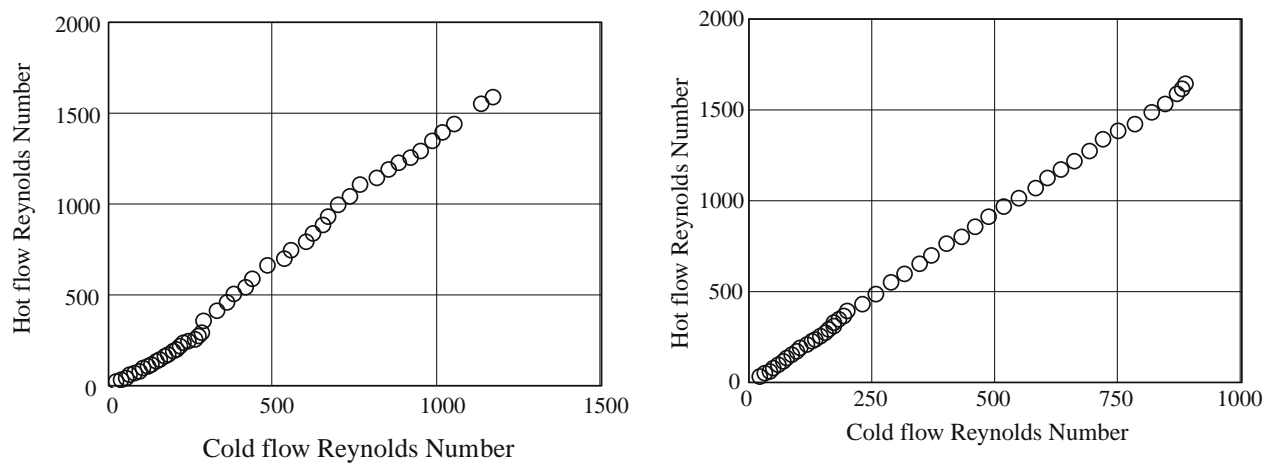

(a) Flow conditions of the experiments
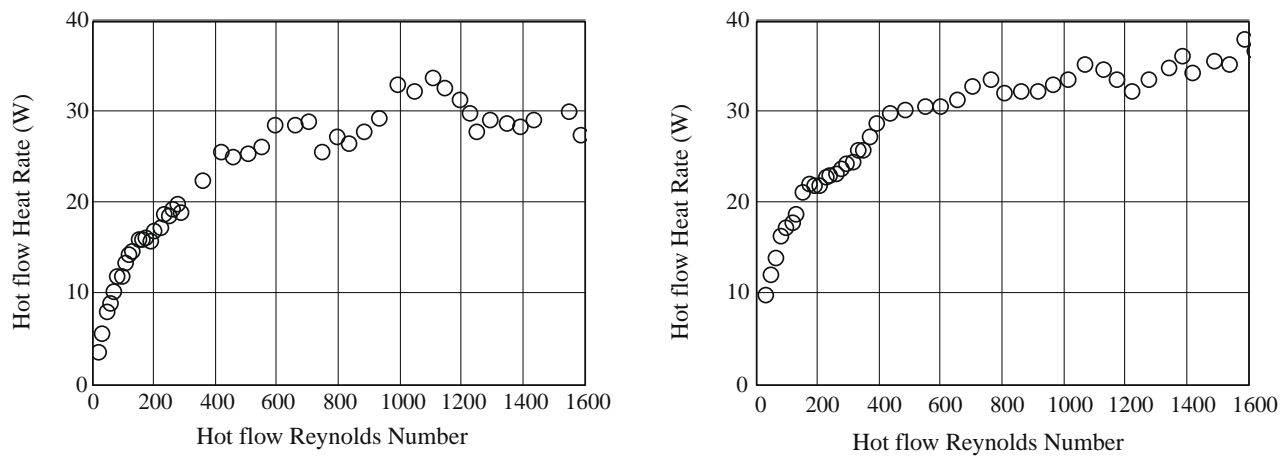

(b) Experimental measurements of the heat transferred from the hot flow
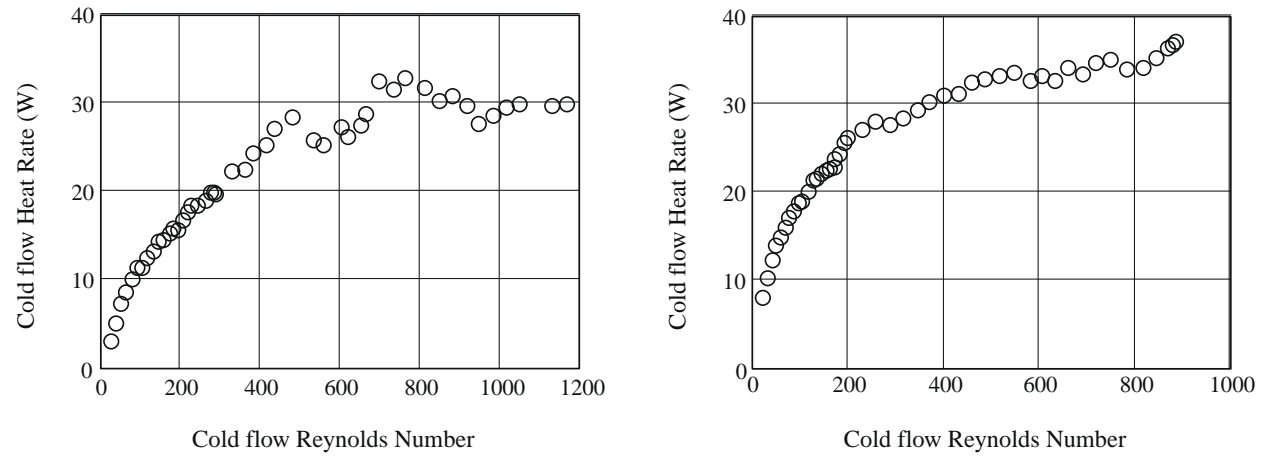

(c) Experimental measurements of the heat transferred to the cold flow
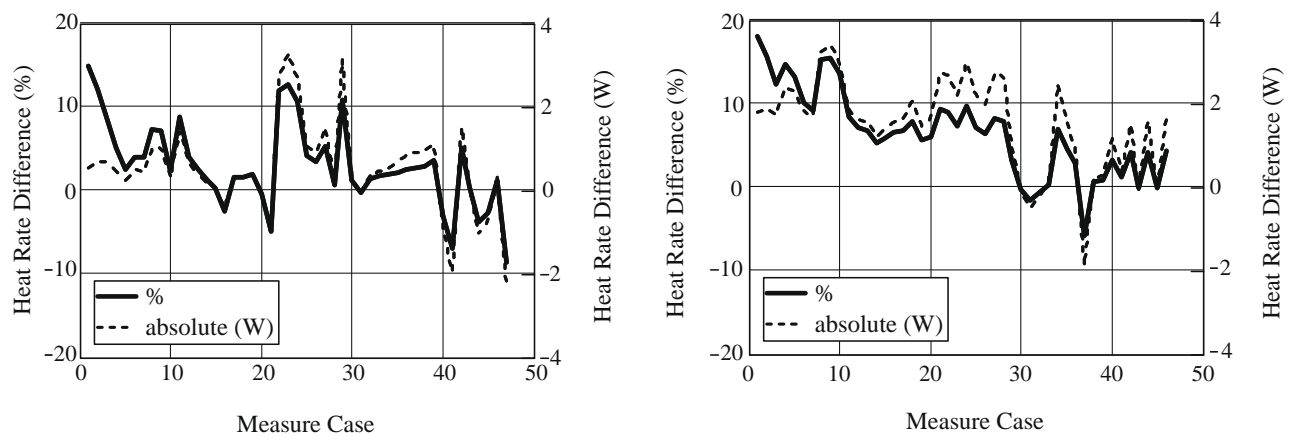

(d) Heat Rate difference

$200 \mu \mathrm{m}$ heat exchanger

$100 \mu \mathrm{m}$ heat exchanger

Fig. 9. Experimental results for both micro-heat exchangers.

The heat rate experimental results obtained from the switched configurations (side A or B with hot or cold flow) are extremely similar in both prototypes, so they are not shown in Fig. 9 and such information will be neglected from now on.

\subsubsection{Thermal effectiveness of the micro heat exchangers}

The overall heat transfer coefficient, $U$, can be easily derived from the experimental data. We will refer it to the area $A_{G}$, which is the projected area of the metallic plate where the microchannels 


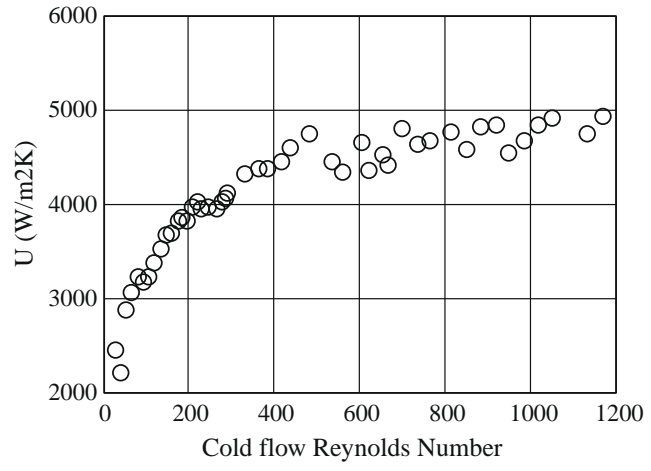

200 microns heat exchanger

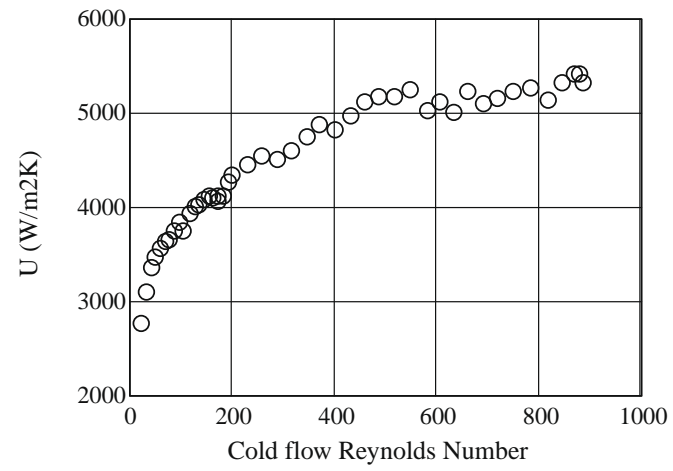

100 microns heat exchanger

Fig. 10. Overall heat transfer coefficients.

have been mechanized ( $20 \mathrm{~mm}$ width $\times 16 \mathrm{~mm}$ length for both prototypes)

$Q \quad U A_{G} \Delta T_{L M} \rightarrow U \quad \frac{Q}{A_{G} \Delta T_{L M}}\left\{\begin{array}{ccc}\Delta T_{L M} & \frac{\Delta T_{1} \Delta T_{2}}{\ln \frac{\Delta T_{1}}{\Delta T_{2}}} \\ \Delta T_{1} & T_{h i} & T_{c o} \\ \Delta T_{2} & T_{h o} & T_{c i}\end{array}\right.$

where the subscript hi represents the hot flow inlet, $c i$ the cold flow inlet, ho the hot flow outlet and co the cold flow outlet. Note that the temperature at the hot flow outlet can be lower than that at the cold flow outlet, as we are using a counterflow heat exchanger.

Fig. 10 shows the overall heat transfer coefficients experimen tally obtained for both heat exchangers. For small Reynolds num bers the heat transfer coefficient increases rapidly with Re, while for larger $\operatorname{Re}(>400)$ it stabilizes in values that are quite similar for both prototypes (slightly higher in the $100 \mu \mathrm{m}$ one).

Employing the classical effectiveness NTU method [26], the mi cro heat exchangers can be characterized, and compared with con ventional large size heat exchangers:

The effectiveness is defined as:

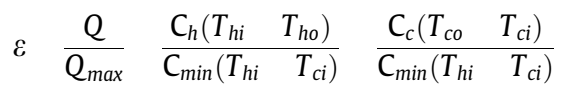

where $C_{c} \quad \dot{m}_{c} c_{c}, C_{h} \quad \dot{m}_{h} c_{h}$ and $C_{\min } \quad \min \left(C_{h}, C_{c}\right)$.

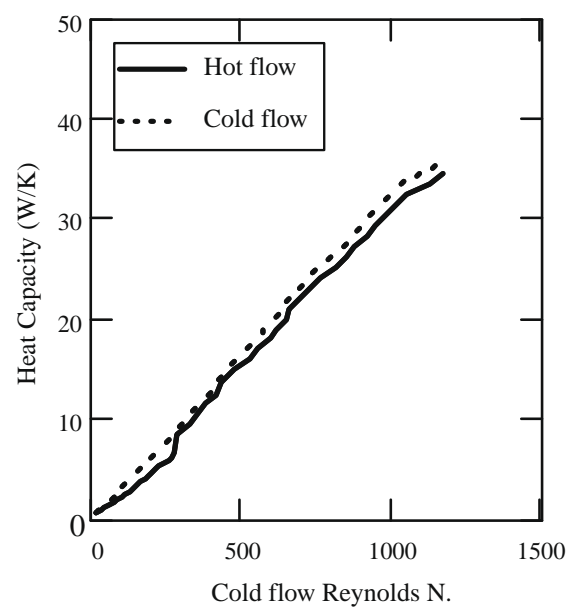

200 microns heat exchanger
Fig. 11 compares the heat capacities, $C_{c}$ and $C_{h}$, of both flows. As it was already pointed out, the pair of mass flows for each experi ment was selected to be similar in both flows, a purpose that was generally attained, with some deviation in the $100 \mu \mathrm{m}$ prototype for large mass flow rates.

The experimental NTU (number of transfer units) of the two prototypes can be obtained following equation:

NTU $\frac{U A_{G}}{\mathrm{C}_{\min }}$

Our results are compared with the values obtained using the analytical expression corresponding to a conventional conterflow heat exchanger showed in Eq. (9) [26]. The data are shown in effec tiveness NTU graphs in Fig. 12. Note that the analytical value of NTU using Eq. (9) is calculated with the experimental values of the effectiveness, and the capacity ratio is evaluated at the average of the measured temperatures. The results show good agreement between analytical and experimental values in both prototypes.

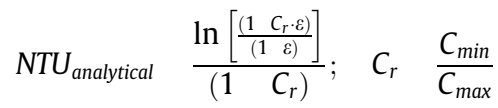

\subsubsection{Average heat transfer coefficients}

The next step of the thermal characterization is to obtain the average convection heat transfer coefficients of the flow in the microchannels. We must remind that the temperature and flow

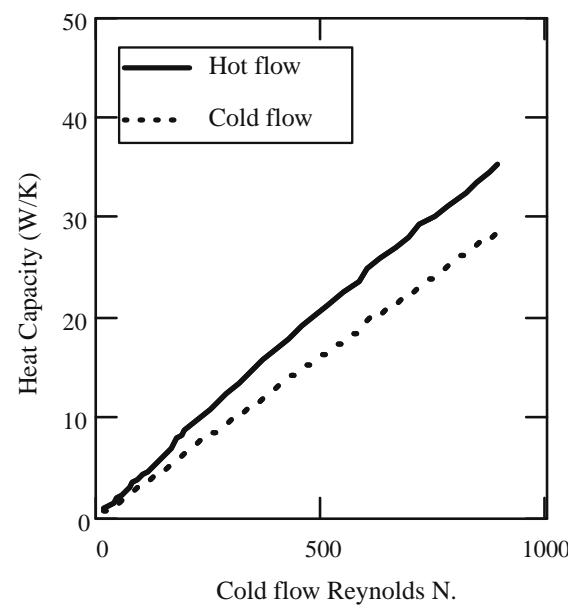

100 microns heat exchanger

Fig. 11. Heat capacities of both flows. 


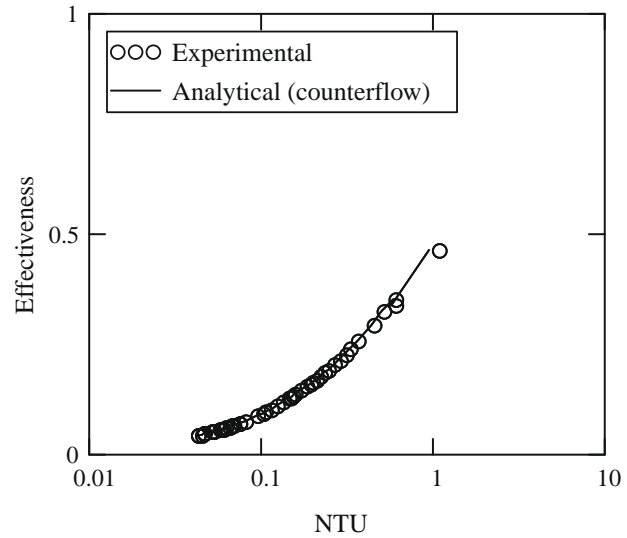

200 microns heat exchanger

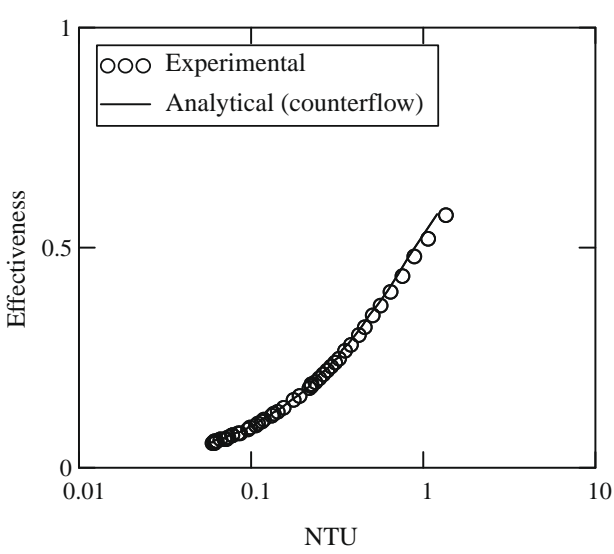

100 microns heat exchanger

Fig. 12. Experimental and analytical NTU comparison.

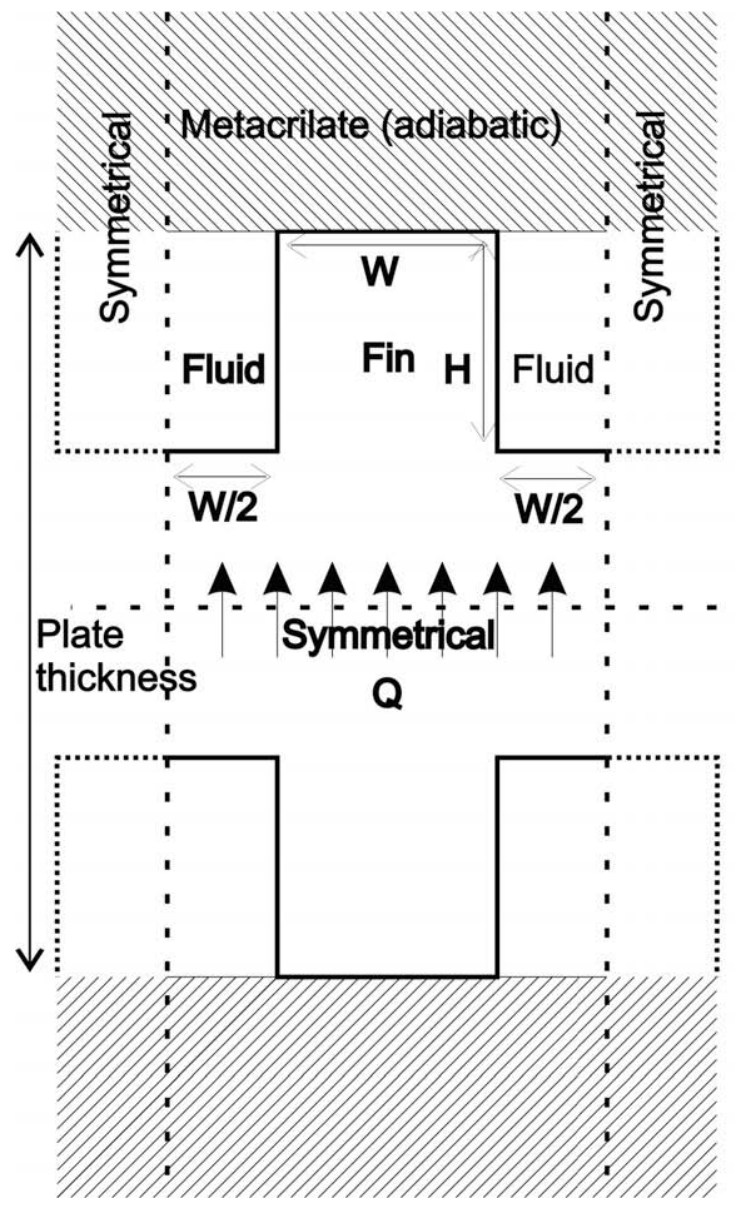

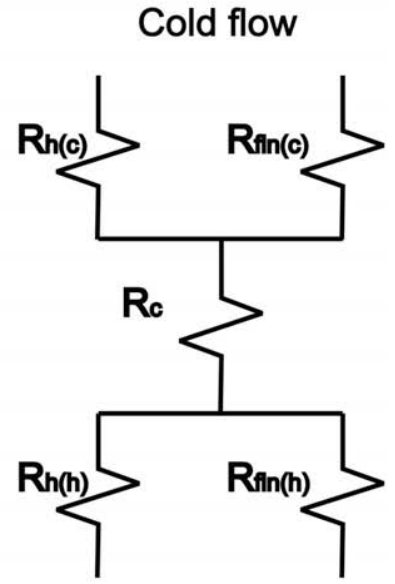

Hot flow

Fig. 13. Microchannel section (out of scale) and equivalent thermal circuit.

measuring systems are located in the prototypes inlet and outlet connections, not inside the microchannels, so the obtained exper imental results can only be used as qualitative information to com pare with the analytical data, and characterize deviations from the predictions of classical theory.

The microchannel geometrical model used in the analysis is showed in Fig. 13. The separation between two adjacent micro channels can be considered an adiabatic tip fin. The thermal resis tance of such a fin is expressed in Eq. (10). The subscript $i$ is used to distinguish between the fins in the cold $(c)$ and in the hot $(h)$ side (while the dimensions are equivalent, the thermal properties vary)

$R_{f i n(i)} \frac{1}{\sqrt{h_{i} P k_{S(i)} A_{C} \tanh \left(\frac{h_{i} P}{k_{S(i)} A_{C}} H\right)}}$

where $\bar{h}_{i}$ is the average heat transfer coefficient of the microchannel flow at the $i$ side, $P$ is the fin perimeter $\left(2 W+2 L_{m}\right), k_{s(i)}$ is the ther 
mal conductivity of the fin material (stainless steel), evaluated at the average temperature of the corresponding plate side, $A_{c}$ is the fin cross sectional area $\left(W L_{m}\right)$ and $H$ is the fin length that in this case is the microchannel depth.

Parallel to the fin resistance, the convection thermal resistance can be written as:

$R_{h(i)} \frac{1}{h_{i} A_{b}}$

where $A_{b}$ is the microchannel bottom area $\left(W L_{m}\right)$.

The conduction thermal resistance corresponding to the stain less steel plate is given in Eq. (12). The thermal conductivity $\left(\bar{k}_{s}\right)$ is evaluated at the average temperature of the steel, which can be obtained from the average temperatures of both fluids. $A$ is the conduction area $(2 W L)$ and $t$ is the thickness (the plate thick ness minus $2 H$ ).

$R_{c} \quad \frac{t}{k_{s} A}$

With Eqs. (10) (12), the total thermal resistance between both flows for the plate element shown in Fig. 13 can be written as:

$R_{T} \quad\left(\frac{1}{R_{f i n(h)}}+\frac{1}{R_{h(h)}}\right)^{1}+R_{c}+\left(\frac{1}{R_{f i n(c)}}+\frac{1}{R_{h(c)}}\right)^{1}$

and the total thermal resistance between flows for the whole plate will be $R_{T} / N$, being $N$ the number of channels in each side of the plate (50 for the $200 \mu \mathrm{m}$ exchanger and 100 for the $100 \mu \mathrm{m}$ one).

Finally, the theoretical overall heat transfer coefficient, $U$, can be obtained as:

$U \frac{1}{A_{G} R_{T}}$

The major parameters in this calculation are the average heat transfer coefficients in both sides of the plate. We have used the same coefficient for Eqs. (10) and (11), but the temperature depen dent variables have been calculated or evaluated separately for the hot and the cold flow sides.

The calculation of the average heat transfer coefficient of the microchannel flow is obtained considering the effect of the en trance region and taking in mind that the microchannels have a square cross section where three sides are heated and the other one is adiabatic. Also, there is an important contraction at the inlet of the microchannels. As Rohsenow et al. [27] sug gested and Lee et al. [28] verified, on such conditions the inlet flow should be assumed as a fully developed flow in the hydro dynamic sense but thermally developing, and so it is assumed in this work.

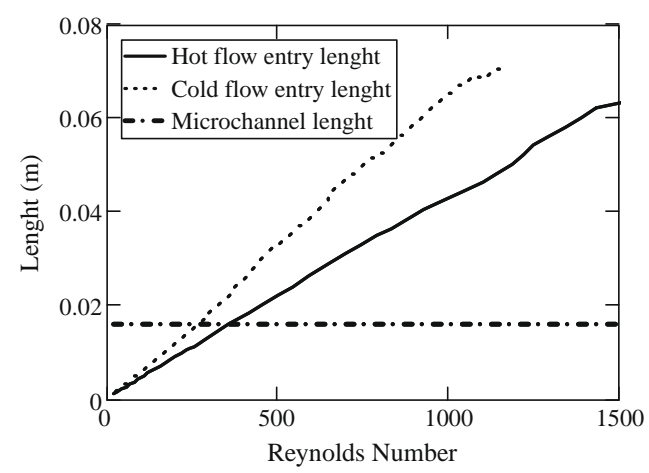

200 microns heat exchanger
The fully developed flow Nusselt number for three sided micro channels, $N u_{f d, 3}$, with an aspect ratio $\left(\alpha_{C} \quad W / H \quad 1\right)$ has a value of 3.556 [21]. Then, the local Nusselt number in the thermal entrance region can be obtained from Eq. (15) [21]

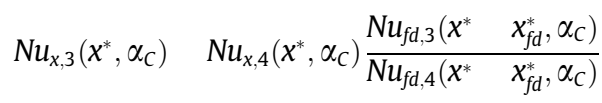

where $x^{*} \quad \frac{x / D_{h}}{\operatorname{RePr}}$ is the non dimensional microchannel length, the subscript $f d$ means fully developed flow, and the subscripts 3 and 4 refer to the three sided and four sided microchannels, respectively. For four sided microchannels, the Nusselt number for fully developed flow is again obtained from Kandlikar et al. [21] (with a value of 3.599) and the local Nusselt number in the entrance region is calculated following Eq. (16) from Lee and Gar imella [29]:

$N u_{x, 4}\left(x^{*}, \alpha_{C}\right) \quad \frac{1}{C_{1}\left(x^{*}\right)^{C_{2}}+C_{3}}+C_{4} \quad 1 \leq \alpha_{C} \leq 10$

where

$$
\begin{array}{lll}
C_{1} & 3.122 \times 10^{3} \alpha_{C}^{3}+2.435 \times 10^{2} \alpha_{C}^{2} \\
& +2.143 \times 10^{1} \alpha_{C}+7.325 \\
C_{2} & 6.412 \times 10^{1} \\
C_{3} & 1.589 \times 10^{4} \alpha_{C}^{2} & 2.603 \times 10^{3} \alpha_{C}+2.444 \times 10^{2} \\
C_{4} & 7.148 \quad 1.328 \times 10^{1} / \alpha_{C}+1.515 \times 10^{1} / \alpha_{C}^{2} & 5.936 / \alpha_{C}^{3}
\end{array}
$$

This expression was obtained analyzing the microchannels heat transfer problem under the $\mathrm{H} 1$ thermal boundary condition (cir cumferentially constant temperature and axially constant heat flux on the walls), which has been found as the best representing boundary condition of the four sided microchannels heat transfer case. Using the Phillips correction factor (Eq. (15)), it can be used as well with three sided microchannels. Nevertheless, it should be noted that this implies that the ratio of developing Nusselt numbers for the three and four sided heating cases is identical to that in fully developing flow [29].

The total length of the entrance region can be calculated from Lee and Garimella [29], where a non dimensional value of 0.062 is considered for channels with an aspect ratio $1\left(\alpha_{C} \quad 1\right)$. Fig. 14 shows the thermal entrance region lengths in our experimental conditions (shown in Fig. 9(a)) as a function of the Reynolds num ber, and compared with the actual microchannel length $(16 \mathrm{~mm})$.

The results show that the entrance region is of paramount importance in both prototypes. The microchannels are completely under entrance region flow conditions even for relatively low Rey nolds numbers. The critical Reynolds numbers (where the entrance length equals the actual length of the channel) are 486 and 760 for

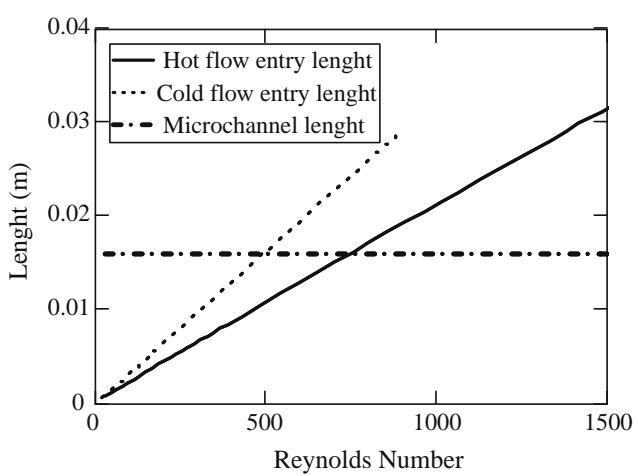

100 microns heat exchanger

Fig. 14. Thermal entrance length. 

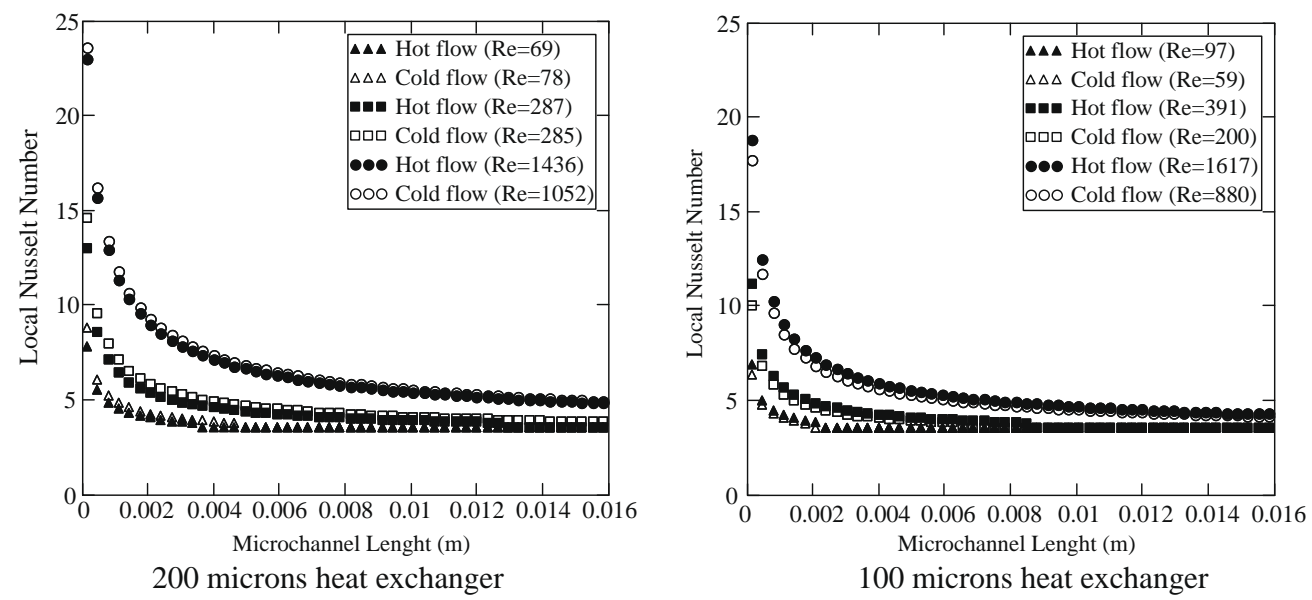

Fig. 15. (Analytical) local Nusselt number in the microchannels.

the cold and hot flow, respectively, in the $100 \mu \mathrm{m}$ exchanger, and 262 and 416 in the $200 \mu \mathrm{m}$ exchanger. Entrance region phenomena would affect an unneglectable portion of the channel, even for very small Reynolds numbers. Therefore, the effect of the entrance re gion could not be obviated.

Fig. 15 shows the Nusselt number obtained with Eqs. (15) and (16) for three of our experimental cases (defined by the pair of Rey nolds numbers corresponding to hot and cold flows) for each pro totype. The large Nusselt numbers represent the incidence of the entrance region, which is more important for higher Reynolds numbers. Note that the properties and conditions in Eq. (15) are evaluated using the experimental results presented before.

The integration of the local Nusselt number over the entire microchannel gives an average Nusselt number. Fig. 16(a) shows this average Nusselt number as a function of the Reynolds number. For Reynolds tending to zero, the average Nusselt number tends to the fully developed value (3.556). As the Reynolds number in creases, the entrance region becomes larger and the average Nus selt number increases. For similar Reynolds numbers, the entrance region is larger for the $200 \mu \mathrm{m}$ microchannels (as shown in Fig. 14) and thus the average Nusselt number is also larger. From these results, the analytical average heat transfer coefficients of both flows can be obtained. Fig. 16(b) shows the results. As already stated, the small differences between hot and cold flows are a con sequence of the temperature based fluid properties variations. As a result of similar Nusselt numbers (especially for low Re), the heat transfer coefficients found in the $100 \mu \mathrm{m}$ microchannels are approximately twice larger than the $200 \mu \mathrm{m}$ ones.

Once the convection coefficients have been obtained, the total resistance for the conditions of each of our experiments can be cal culated following Eq. (13). Fig. 17 shows the results, distinguishing between the global convection resistance in each side and the con duction resistance. The conduction term is often neglected in large scale heat exchangers, but it is of importance in micro heat exchangers, where convection coefficients are extremely large and the fabrication procedure restricts the plate material and the minimum plate thickness.

Fig. 17 shows that, for the chosen experimental conditions, both convection resistances are very similar and smaller than the con duction resistance. Nevertheless, the convection resistances cannot be neglected and even have a major influence, especially in the $200 \mu \mathrm{m}$ exchanger and for low Reynolds numbers, where they amount to half the global resistance. While the convection resis tances are larger for decreasing Reynolds number, the conduction

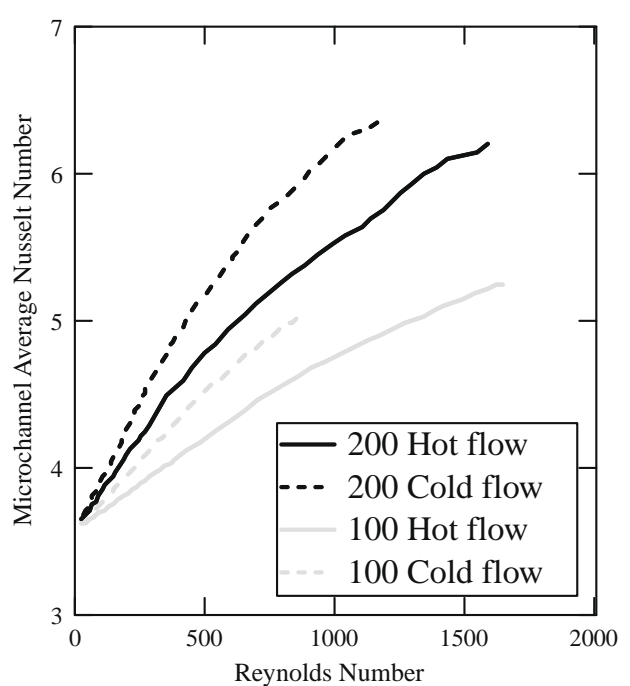

(a) Nusselt number

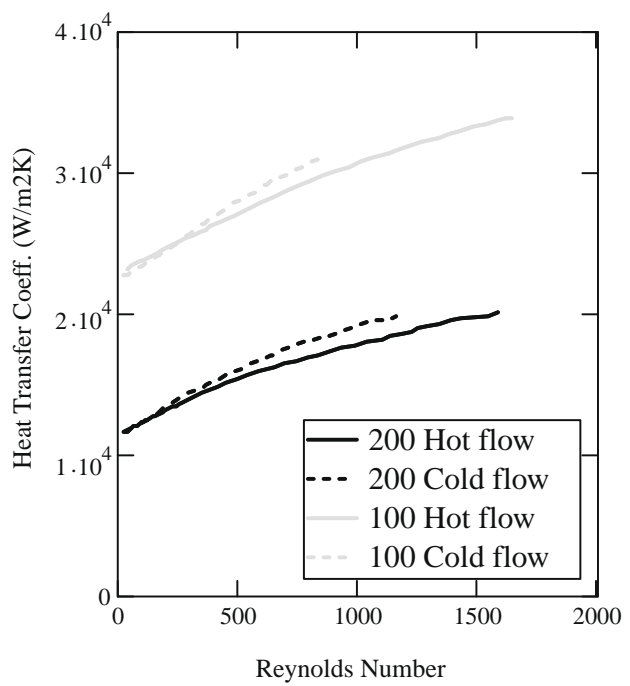

(b) Convection heat transfer coefficient

Fig. 16. Average Nusselt number and convection coefficient in the microchannels. 


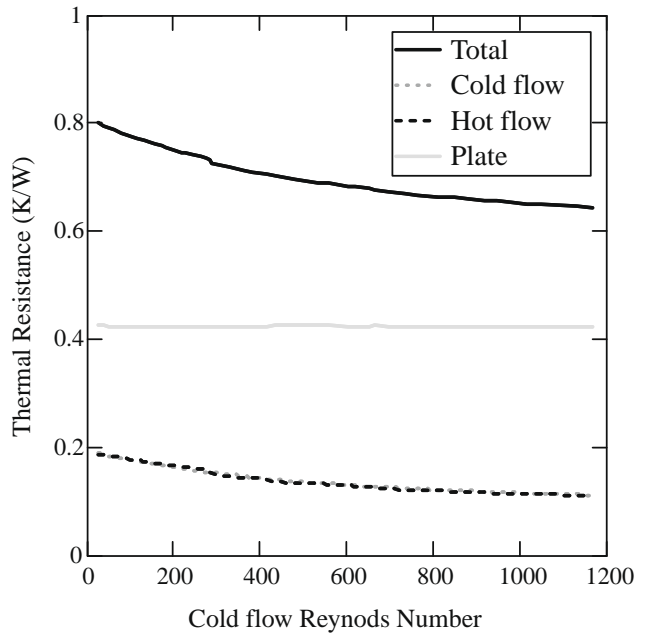

200 microns heat exchanger

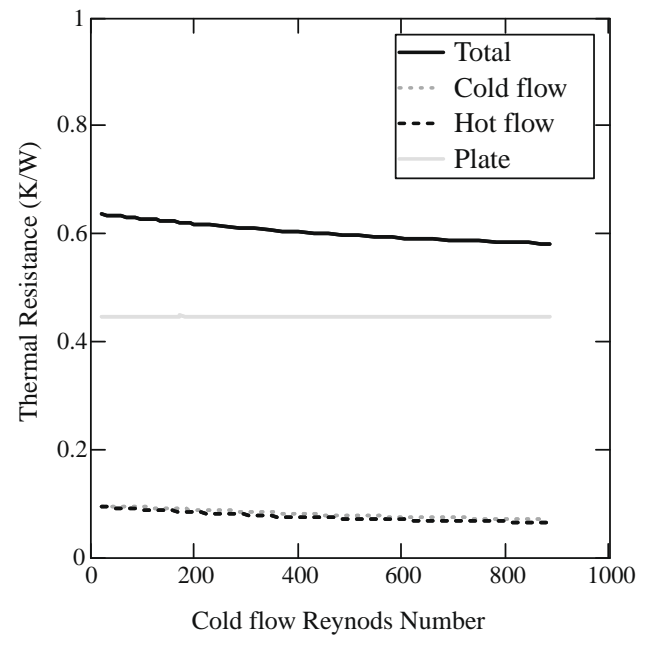

100 microns heat exchanger

Fig. 17. Comparison of the three thermal resistances in Eq. (13).

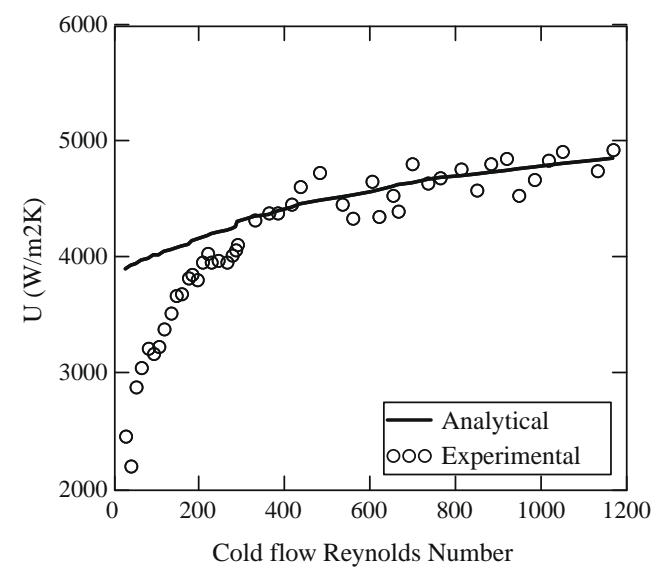

200 microns heat exchanger

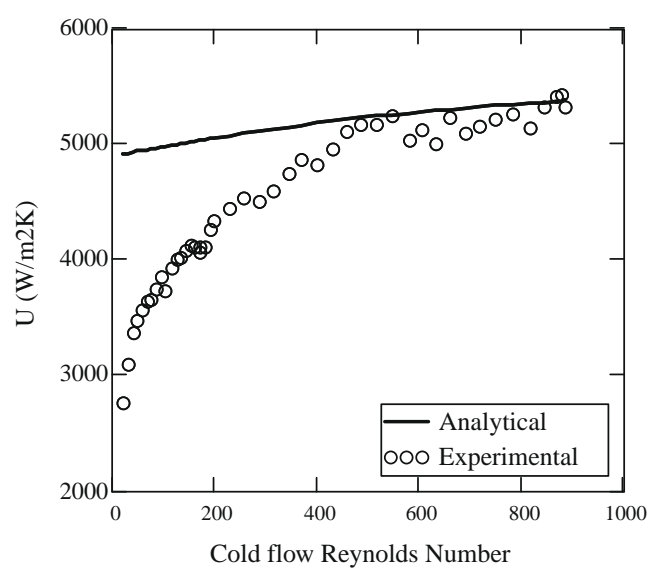

100 microns heat exchanger

Fig. 18. Experimental and analytical overall heat transfer coefficients.

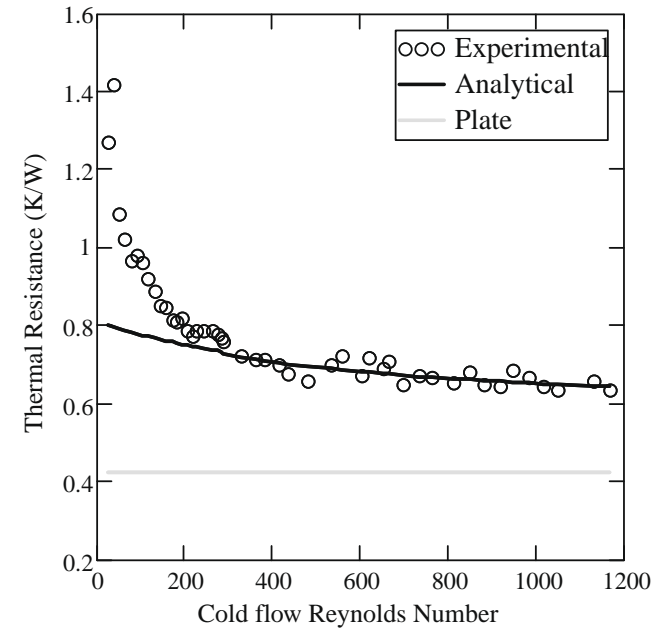

200 microns heat exchanger

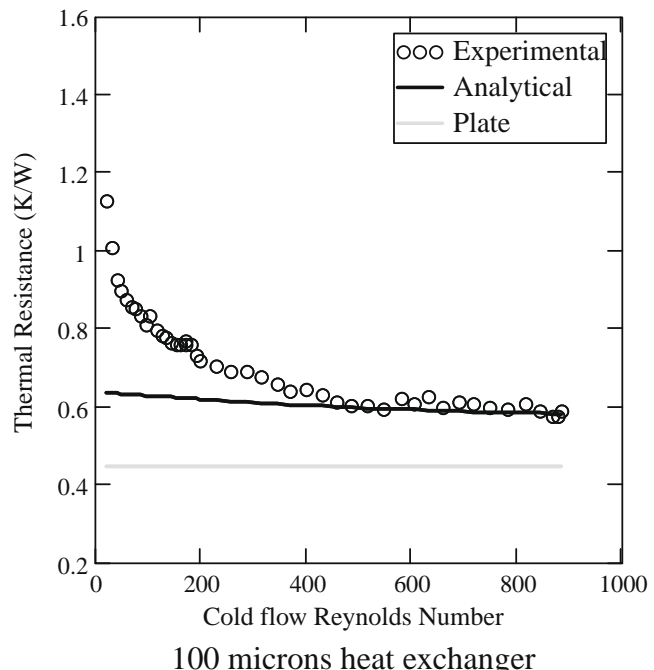

100 microns heat exchanger

Fig. 19. Total thermal resistances and plate conduction resistance. 
resistance remains almost constant, as the mean temperature vari ations of the plate for the different experimental conditions are small and thus produce negligible heat conductivity variations.

Although this paper focuses on the convection effects, the con duction resistance has an obvious relevance. Therefore, the plate thickness and plate material are critical design parameters. Fabri cation processes for channel mechanization must allow smaller thicknesses (around $1 \mathrm{~mm}$ or less) or use materials with higher conductivity (around $100 \mathrm{~W} / \mathrm{mK}$ or higher).

From these data, the overall heat transfer can be immediately obtained. A comparison with the experimental values already shown in Fig. 10 is straightforward. Fig. 18 shows the results for the conditions of the experiments (again defined by the cold flow Reynolds number), and the comparison with the experimental data.

In both exchangers the analytical values of $U$ are in agreement with the experimental results for large Reynolds Numbers, but there are strong discrepancies for small Re. This last region seems to be larger for the $100 \mu \mathrm{m}$ exchanger $(\mathrm{Re}<400$ for the $100 \mu \mathrm{m}$ ex changer and $\operatorname{Re}<200$ for the $200 \mu \mathrm{m}$ exchanger). This behavior may be easierly understood if we look at the thermal resistances shown in Fig. 19.

The graphs of Fig. 19 show a comparison between the analytical and the experimental total thermal resistances. The conduction ef fect, also shown is the graphs can be subtracted from both resis tances (although it has not been obtained experimentally, no huge variations can be expected). Then, the information regards exclusively to the convection effects in both sides.

For low Reynolds numbers the analytical expression seems to underestimate the thermal resistance, and thus the calculation overestimates the convection coefficient. It should be reminded that the Nusselt number tends to the fully developed value for low Reynolds number. Therefore, a smaller value of the convection coefficient neither can be related to entrance region effects due to surface roughness or other causes, nor to any turbulent effect. A major source of errors may be the boundary condition used in the analysis. The $\mathrm{H} 1$ condition might not be adequate at low Re, considering small temperature variations along the channel and the incidence of phenomena related to the square, three sided geometry of the channels, as stated by Kays and Crawford [30]. Moreover, and as was stated in Section 3.2.1 and shown in Fig. 9, the heat losses at low Re conditions demand particular attention because their value becomes important compared with the heat effectively transferred between fluids. It should be noted that the correlations used in this work have been compared by their authors (Lee and Garimella [29]) only with experimental data that has been obtained at higher Reynolds numbers (larger than 300).

Finally, for Reynolds larger than 200 400, the experimental re sults fit well with the analytical ones. Therefore, the Nusselt num ber analytical expressions, based on a classical, continuum approach, seem adequate to predict heat transfer coefficients in microchannels of $100200 \mu \mathrm{m}$.

\section{Concluding remarks}

In this work, we have presented an experimental study of the hydrodynamic and heat transfer performance of micro heat exchangers without phase change. Two prototypes were designed and characterized, with microchannel of dimensions of 100 and $200 \mu \mathrm{m}$. The results show good agreement with the general theory and no special effect related to the small dimension of the channels was observed.

The fluid pressure drop along the micro heat exchangers was measured for a wide range of (laminar) Reynolds numbers. The results adjust to the predictions of conventional laminar flow the ory for both prototypes.

The heat transfer performance was studied and compared with conventional heat transfer theory, applicable to larger devices. No heat transfer increment appeared as a result of the small channel dimensions. The entrance region phenomena define the heat trans fer for medium and large Reynolds number and this region is well predicted by correlations existing in the literature. For very low Reynolds numbers, the results show discrepancies with the avail able models and correlations, which overestimate the convection coefficients. This seems to be related with the adequacy of the boundary conditions. On the other hand, the plate conduction ther mal resistance is a major restriction for micro heat exchangers per formance. The plate thickness and plate material are critical in the design of micro heat exchangers.

\section{References}

11] S.S. Mehendale, A.M. Jacobi, R.K. Shah, Fluid flow and feat transfer at micro and meso-scales with applications to heat exchanger design, Appl. Mech. Rev. 53 (2000) 175-193.

[2] S.G. Kandlikar, W.J. Grande, Evolution of microchannel flow passages thermohydraulic performance and fabrication technology, Heat Transfer Eng. 24 (1) (2003) 3-17

[3] D.B. Tuckermann, R.F.W. Pease, High performance heat sinking for VLSI, IEEE Electron Device Lett. 2 (5) (1981) 126-129.

[4] P. Wu, W.A. Little, Measurement of friction factors for the flow of gases in very fine channels used for microminiature Joule-Thomson refrigerators, Cryogenics 23 (5) (1983) 273-277.

[5] G.L. Morini, Single-phase convective heat transfer in microchannels: a review of experimental results, Int. J. Therm. Sci. 43 (2004) 631-651.

[6] M.E. Steinke, S.G. Kandlikar, Review of single-phase liquid heat transfer in microchannels, in: ASME, Proceedings of the Third International Conference on Microchannels and Minichannels, Toronto, ON, Canada, Paper ICMM200575114,2005

[7] M.J. Kohl, S.I. Abdel-Khalik, S.M. Jeter, D.L. Sadowski, An experimental investigation of microchannel flow with internal pressure measurements, Int. J. Heat Mass Transfer 48 (2005) 1518-1533.

[8] S. Chakraborty, K.D. Anand, Implications of hydrophobic interactions and consequent apparent slip phenomenon on the entrance region transport of liquids through microchannels, Phys. Fluids 20 (043602) (2008) 1-9.

[9] S. Chakraborty, Order parameter modeling of fluid dynamics in narrow confinements subjected to hydrophobic interactions, Phys. Rev. Lett. 99 (094504) (2007) 1-4.

[10] S. Chakraborty, Towards a generalized representation of surface effects on pressure-driven liquid flow in microchannels, Appl. Phys. Lett. 90 (034108) (2007) 1-3.

[11] S.M. Ghiaasiaan, T.S. Laker, Turbulent forced convection in microtubes, Int. J. Heat Mass Transfer 44 (2001) 2777-2782.

[12] I. Papautsky, J. Brazzle, T. Ameel, A.B. Frazier, Laminar fluid behavior in microchannels using micropolar fluid theory, Sens. Actuators 73 (1999) 101108.

[13] C.P. Tso, S.P. Mahulikar, The use of the Brinkman number for single phase forced convective heat transfer in microchannels, Int. J. Heat Mass Transfer 41 (12) (1998) 1759-1769.

[14] C.P. Tso, S.P. Mahulikar, The role of the Brinkman number in analysing flow transitions in microchannels, Int. J. Heat Mass Transfer 42 (1999) 1813-1833.

[15] C.P. Tso, S.P. Mahulikar, Experimental verification of the role of Brinkman number in microchannels using local parameters, Int. J. Heat Mass Transfer 43 (2000) 1837-1849.

[16] G.M. Mala, D. Li, J.D. Dale, Heat transfer and fluid flow in microchannels, Int. J. Heat Mass Transfer 40 (13) (1997) 3079-3088.

[17] C. Yang, D. Li, Electrokinetic effects on pressure-driven liquid flows in rectangular microchannels, J. Colloid Interface Sci. 194 (1997) 95-107.

[18] C. Yang, D. Li, Analysis of electrokinetic effects on the liquid flow in rectangular microchannels, Colloids Surf. A 143 (1998) 339-353.

[19] D. Li, Electro-viscous effects on pressure-driven liquid flow in microchannels, Colloids Surf. A 195 (2001) 35-57.

[20] S. Chakraborty, T. Das, S. Chattoraj, A generalized model for probing frictional characteristics of pressure-driven liquid microflows, J. Appl. Phys. 102 (104907) (2007) 1-11.

[21] S.G. Kandlikar, S. Garimella, D. Li, S. Colin, M.R. King. Heat Transfer and Fluid Flow in Minichannels and Microchannels, first ed., Elsevier, 2006. pp. 87-135.

[22] F. Abdelall, G. Hans, S.M. Ghiaasiaan, S.I. Abdel-Khalik, S.M. Jeter, M. Yoda, D. Sadowski, Pressure drop caused by abrupt flow area changes in small channels, Exp. Therm. Fluid Sci. 29 (4) (2005) 425-434.

[23] S. Kakac, R.K. Shah, W. Aung, Handbook of Single-Phase Convective Heat Transfer, John Wiley and Sons, New York, 1987

[24] M.E. Steinke, S.G. Kandlikar, Single-phase liquid friction factor in microchannels, Int. J. Therm. Sci. 45 (2006) 1073-1083. 
[25] J. Li, G.P. Peterson, P. Cheng, Three-dimensional analysis of heat transfer in a micro-heat sink with single phase flow, Int. J. Heat Mass Transfer 47 (2004) 4215-4231.

[26] G.F. Hewitt, G.L. Shires, T.R. Bott, Process Heat Transfer, CRC Press, 1994. pp. 155-194.

[27] W.M. Rohsenow, J.P. Hartnett, E.N. Ganic, Handbook of Heat Transfer Applications, McGraw-Hill, New York, 1985.
28] P.S. Lee, S.V. Garimella, D. Liu, Investigation of heat transfer in rectangular microchannels, Int. J. Heat Mass Transfer 48 (2005) 1688-1704.

[29] P.-S. Lee, S.V. Garimella, Thermally developing flow and heat transfer in rectangular microchannels of different aspect ratios, Int. J. Heat Mass Transfer 49 (2006) 3060-3067.

[30] W.M. Kays, M.E. Crawford, Convective Heat and Mass Transfer, third ed., McGraw-Hill, 1993. pp. 108-158. 